\title{
Cochonilha rosada, maconellicoccus hirsutus (green): Uma praga de importância quarentenária já se encontra na guiana inglesa
}

\author{
IFernando Junqueira Tambasco \\ iLuiz Alexandre Nogueira de Sá \\ iElzabeth Aparecida Baptista de Nardo \\ ${ }_{2}$ Marcelo Teixeira Tavares
}

Palavras chave: Maconellicoccus hirsutus, Cochonilha rosada, praga quarentenária $\mathrm{A} 1$

\section{INTRODUÇÃo}

A cochonilha rosada, Maconellicoccus hirsutus (Green) (Hemiptera, Homoptera: Pseudococcidae), é considerada praga quarentenária para o Brasil, já ocorrendo em vários países do mundo. Em 1994 invadiu as ilhas do Caribe disseminando-se rapidamente para mais de 14 países daquela região. Em 1997, foi detectada pela primeira vez no continente sul-americano, na Guiana Inglesa (USDA 1997). Baseado no histórico de sua rápida disseminação no Caribe, é atualmente aceito que a entrada desse inseto nos países fronteiriços, como o Brasil, é somente uma questão de tempo, tendo em vista a proximidade de nosso país com a Guiana Inglesa. Além do mais, o Brasil oferece condições climáticas favoráveis ao estabelecimento e desenvolvimento da praga, conforme previsto pelo software CLIMEX, utilizado em análise-de-risco de pragas (MACONELLICOCCUS, 1998).

Por definição, uma praga quarentenária é um organismo de importância econômica potencial para a área posta em perigo e onde ainda não está presente (BRASIL, 1995). A Portaria nº 181, de 05 de Outubro de 1998, declara alerta máximo a estas pragas quarentenárias A1, ainda não presentes no país, como é o caso da cochonilha rosada.

O impacto econômico esperado pela entrada da cochonilha rosada, sobre a agricultura brasileira, é grande, pois trata-se de uma espécie polífaga, já detectada em mais de 200 espécies de plantas no mundo, muitas delas de

'Embrapa Meio Ambiente, CP 69 Jaguariúna, SP, Fone: 193867 8791, lqcl-1@ cnpma.embrapa.br. , ${ }^{2}$ Depto. Ciências Exatas e Naturais, UNIARA, Araraquara, SP, Fone: 162220499

biodiversidade@uniara.com.br 
importância econômica para o país, tais como: algodão, citros, cacau, café, graviola, e uva. Para as ornamentais como o hibisco, em parques e jardins de residências, causa sérios danos de valor estético. O impacto negativo da introdução de pragas em áreas isentas deve ser considerado não somente do ponto de vista econômico, mas também ambiental, devido ao efeito que as medidas de controle adotadas contra a nova praga possam ter sobre os recursos naturais, organismos não visados e as competições biológicas com as espécies nativas (KOGAN 1997). As espécies de plantas mais suscetíveis encontram-se nas famílias Malvaceae, Fabaceae, Moraceae, Proteaceae e Rhamnaceae (SILVA, 1997).

\section{INFORMAÇÕES SOBRE A PRAGA}

Maconellicoccus hirsutus (Green) pertence à família Pseudococcidae e é conhecida como cochonilha rosada do hibisco "pink hibiscus mealybug", pela sua coloração e associação constante com esta planta ornamental, ou "grape mealybug", pelo severo dano que causa a plantas do gênero Vitis (CHANG \& MILLER 1996). Existe atualmente um esforço internacional no sentido de padronizar o nome da praga para "pink hibiscus mealybug", mesmo sabendo que ela ataca muitas outras espécies de plantas. A cochonilha rosada foi originalmente descrita na Índia, em 1908, e introduzida, também naquele ano, no Egito, de onde se espalhou para muitas partes da África Tropical. Tornou-se uma praga séria de muitas plantas e atualmente pode ser encontrada na Austrália e Ilhas do Pacífico, Ásia, Oriente Médio, África, Antilhas e Norte da América do Sul (NARDO et al., 1999).

A cochonilha rosada suga a seiva do hospedeiro e injeta-lhe uma saliva tóxica enquanto está se alimentando. Este processo leva à malformação das folhas e frutos, assim como ao crescimento apical encarquilhado, podendo causar diretamente a morte das plantas. As flores infestadas secam e caem, e frutos não são produzidos. Frutos infestados são menores, de formato anormal, podendo cair precocemente, reduzindo, assim a produção e seu valor comercial (NARDO et al., 1999)

A fêmea adulta mede cerca de $3 \mathrm{~mm}$ de comprimento e é áptera, com uma camada branca, floculada, cobrindo a superfície dorsal e apresenta dois filamentos cerosos caudais muito pequenos e filamentos laterais ausentes. $\mathrm{O}$ corpo da fêmea e a hemolinfa são rosados e esta secreta uma substância semelhante a algodão de forma irregular (ovissaco) onde coloca de 300 a 600 ovos, e as ninfas de primeiro estágio são móveis. Em clima tropical a cochonilha-rosada leva de 23 a 30 dias para completar seu ciclo de vida. Os machos passam por quatro estágios de ninfa, são menores que as fêmeas, alaranjados, têm um par de asas e dois filamentos caudais cerosos, não se alimentam e vivem por apenas alguns dias, até acasalarem. As fêmeas virgens 
produzem feromôneo sexual que atrai os machos para a cópula e completam o ciclo até a fase adulta passando por três estágios de ninfa. As cochonilhas podem se dispersar pelo vento, ou as ninfas de primeiro estágio podem se dispersar andando a procura de plantas hospedeiras. Em regiões mais frias os ovos da cochonilha rosada podem passar o inverno abrigados em ranhuras nas cascas das árvores ou no solo (STIBICK 1997).

A Figura 1 referente ao ciclo biológico da cochonilha rosada encontra-se no final do texto

\section{CONTROLE}

Os métodos de controle tradicional como o Controle Químico, Controle Cultural e Controle Físico dificilmente são bem sucedidos no controle deste inseto, uma vez que ele vive em condições protegidas nas plantas, em fendas, rachaduras de cascas de árvores e pecíolos, além de apresentar uma grossa camada cerosa no corpo e seus ovos serem protegidos em um ovissaco, dificultando o acesso e penetração de inseticidas. Assim, a aplicação de inseticida ou a poda drástica e queima do material são técnicas ineficientes no controle da cochonilha rosada, como foi evidenciado em vários países da região do Caribe (PERSAD, 1998). A alternativa de controle mais viável é o controle biológico, com a utilização de parasitóides e predadores conforme resultados observados nos programas que estão sendo conduzidos nesses países (KAIRO et al., 1998; MEYERDIRK et al., 1998; THOMAS et al., 1998; USDA, 1997 )

Desde a entrada de $M$. hirsutus na região do Caribe, esforços começaram a ser direcionados no sentido de iniciar programas de controle biológico. Várias instituições internacionais e nacionais iniciaram projetos, incluindo a Food and Agricultural Organization (FAO), o International Institute of Biological Control (IIBC), atualmente denominado CABI-BIOSCIENCE, o Instituto Caribenho de Pesquisa e Desenvolvimento (CARDI), Departamento de Agricultura dos Estados Unidos (USDA, APHIS) e o Ministério da Agricultura e Recursos Terrestres e Marinhos do Caribe (NARDO et al., 1999). Os principais inimigos naturais que estão sendo usados, atualmente, na região do Caribe são os parasitóides Anagyrus kamali e Gyranusoidea indica e os predadores, Cryptolaemus montrouzieri e Scymnus coccivora, (USDA, 1998). Estes insetos estão sendo criados e liberados nos diferentes países do Caribe e na Guiana Inglesa, mostrando eficiência no controle desta praga. Destas quatro 
espécies, A.. kamali e $C$. montrouzieri são as duas espécies mais promissoras e a base de todos os programas de controle biológico daquela região.

Medidas preventivas para reduzir o risco acidental de entrada

O Serviço de Defesa Fitossanitária do Ministério da Agricultura e do Abastecimento (MA-SDV), preocupado com a eventual entrada desta praga no Brasil, preparou um plano emergencial para evitar ou retardar sua introdução Este tempo deve ser usado para preparação de procedimentos apropriados para solucionar o problema quando o inseto aqui chegar.

Quanto ao programa de controle biológico, o MA e a Embrapa Meio Ambiente estabeleceram parceria com instituições internacionais, como o USDA-APHIS e CABI-BIOSCIENCE, para o envio do parasitóide A. kamali, quando necessário, para darmos início ao programa de controle biológico no Brasil. No caso dos predadores, o C. montrouzieri já foi re-introduzido no Brasil recentemente e o Laboratório de Quarentena Costa Lima, na Embrapa Meio Ambiente, mantém colônia deste predador sobre outro hospedeiro.

As medidas para redução de risco de entrada podem ser amplamente categorizadas com a conscientização da população e medidas de quarentena. Aquelas de conscientização da população envolvem campanhas educacionais (vídeos, jornais, panfletos, cartões de identificação da praga, cartazes) elaboradas e dirigidas aos diferentes setores da população, sobre os riscos que estão associados quando se traz plantas e/ou outros organismos de outros países, com específica referência à cochonilha rosada e sua conhecida distribuição no âmbito mundial. A participação da população, desde o início das campanhas de alerta quarentenário, é de extrema importância para o sucesso posterior de qualquer programa de controle que se fizer necessário.

As medidas de quarentena incluem: 1) treinamento de fitossanitaristas do Ministério da Agricultura e do Abastecimento (MA), que atuam nos portos de entrada, para reconhecer produtos agrícolas que podem estar infestados com a cochonilha rosada e aplicação dos procedimentos para com importadores suspeitos ou confirmados; 2) o MA e os fiscais devem dispor de informações atualizadas sobre a dispersão da cochonilha rosada nas diferentes regiões, para poderem avaliar os riscos apresentados pelos meios de disseminação de diferentes lugares; e 3) aumento da inspeção dos produtos agrícolas importados, especialmente daqueles provenientes de áreas infestadas.

Uma campanha de conscientização pública já está elaborada desde o início de 1999, com a confecção de folders, panfletos e pôster. Dois vídeos estão sendo editados e serão veiculados por todo o país, com ênfase para a população que viaja para áreas infestadas. Um deles é um vídeo de alerta quarentenário para ser passado em aeronaves para os passageiros que estão viajando para as regiões contaminadas por esta praga. 
Uma rápida aplicação do controle biológico nos territórios afetados pode diminuir substancialmente a taxa de dispersão para outras regiões e mesmo países. Entretanto, havendo territórios altamente infestados, haverá, inevitavelmente, continuação da disseminação da cochonilha rosada.

Baseando-se no histórico de dispersão rápida da cochonilha rosada, dos danos por ela ocasionados, da viabilidade e sucesso da realização de programas de controle biológico para combatê-la, o governo brasileiro, através do MA, do SDA e do DDIV em cooperação com a Embrapa Meio Ambiente, está direcionando ações para o início imediato de um programa preventivo de controle biológico da cochonilha rosada. $\mathrm{O}$ objetivo é reunir o maior número de informações e dispor de inimigos naturais, técnicos treinados e laboratório de quarentena preparado, para iniciar o controle biológico rapidamente. Algumas das ações iniciadas são: (a) participar de maneira cooperativa nos programas de controle biológico internacionais, principalmente naqueles em desenvolvimento no momento na região do Caribe, com a cooperação da FAO, IIBC (CABI BIOSCIENSE) e USDA (APHIS), cujos países participantes têm direito a qualquer inimigo natural coletado, os resultados são compilados e disponibilizados aos participantes em uma rede cooperativa. O objetivo é otimizar os recursos financeiros e de pessoal. Nesse sentido, o Laboratório de Quarentena "Costa Lima" estabeleceu contatos com pesquisadores do APHISUSDA e do IIBC, os quais se prontificaram a enviar os inimigos naturais tão logo o Brasil os solicite; (b) iniciar atividades de levantamento da existência de um possível complexo nativo de inimigos naturais da cochonilha-rosada, através de coleta e identificação de inimigos naturais existentes em pseudococcídeos presentes no Brasil e que pertençam a gêneros próximos ao da cochonilha rosada. Os inimigos encontrados e identificados poderiam ser criados em laboratório e testados quanto à especificidade/eficiência em controlar a cochonilha rosada. Tais testes poderão ser realizados no exterior, onde a praga já ocorre. Nesse caso, remessas dos possíveis inimigos naturais identificados deveriam ser feitas para o local desejado; essa é uma área de pesquisa aberta, cujos estudos poderão ser concentrados. Um levantamento bibliográfico dos inimigos naturais da cochonilha rosada, presentes no Brasil, já foi iniciado pela equipe do Laboratório de Quarentena (NARDO et al. 1999); (c) estimular e suportar, financeiramente, projetos de controle biológico e capacitação de recursos humanos em programas de controle biológico da cochonilha rosada, através da organização e participação em cursos/viagens e recebimento de consultorias.

Outras medidas que já foram ou estão sendo implementadas no Brasil são: campanhas públicas de alerta à população e pesquisadores em geral por meio de palestras, confecção e distribuição de folderes, pôsteres e outros tipos de publicações e pela divulgação na mídia através de entrevistas, artigos de jornais, e outros meio de comunicação (ALERTA, $1998 a, b$ e $c$; NARDO \& TAMBASCO, 1998; TAMBASCO \& NARDO, $1998 a$ e $b$; TAMBASCO, 
1998), treinamento de inspetores e técnicos pertencentes ao MA no reconhecimento da praga e no conhecimento do programa de controle biológico implantado em outros países.

Figura 1 - Ciclo biológico da cochonilha rosada (Adaptado de USDA, 1998)

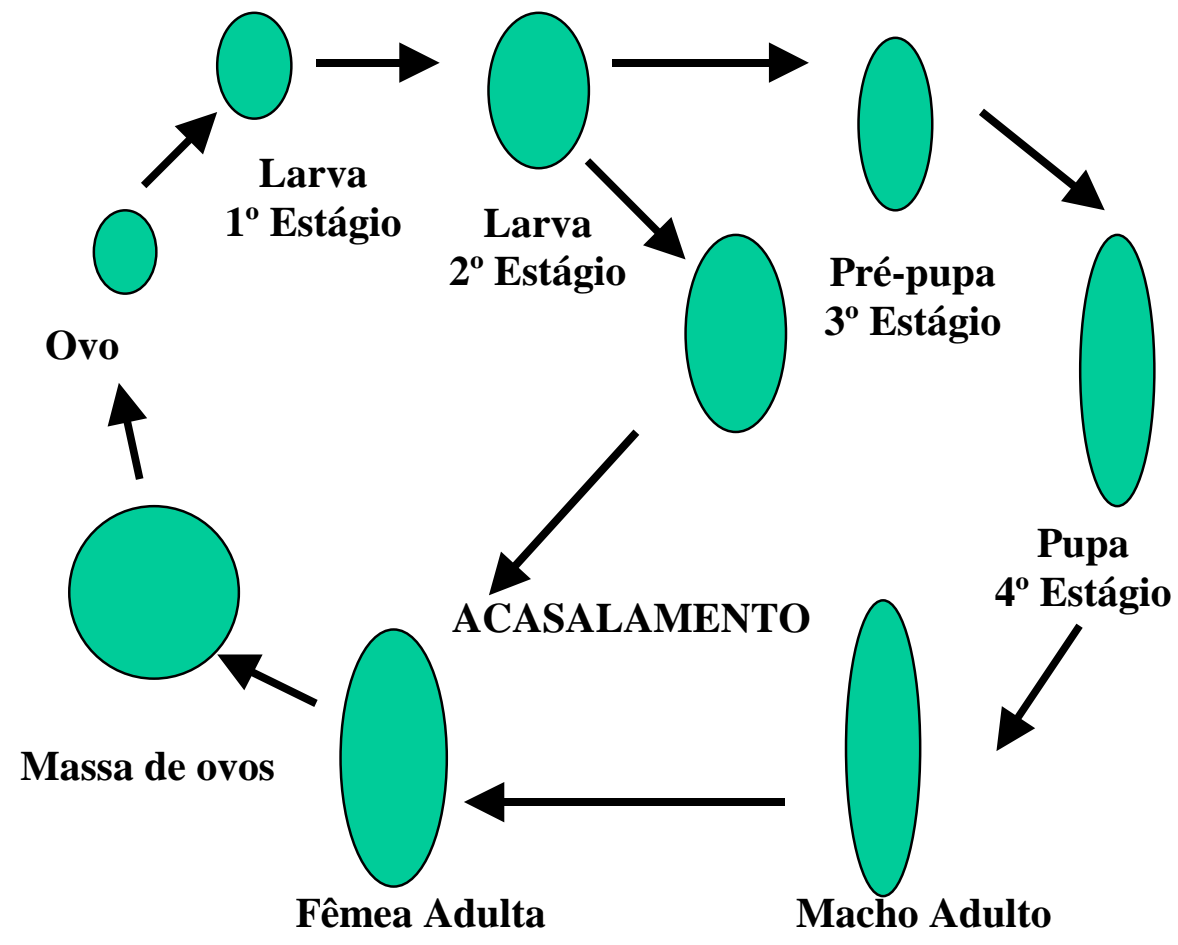




\section{REFERÊNCIAS BIBLIOGRÁFICAS}

ALERTA quarentenário: atenção para a cochonilha rosada. Jaguariúna: Embrapa Meio Ambiente - Laboratório de Quarentena "Costa Lima"/Brasília: Ministério da Agricultura e do Abastecimento, 1998a. Folder.

ALERTA quarentenário: atenção para os sintomas de ataque da cochonilha rosada. Jaguariúna: Embrapa Meio Ambiente - Laboratório de Quarentena "Costa Lima"/Brasília: Ministério da Agricultura e do Abastecimento, 1998b. Cartaz.

ALERTA quarentenário: cochonilha rosada, Maconellicoccus hirsutus (Green). Jaguariúna: Embrapa Meio Ambiente/Brasília: Ministério da Agricultura e do Abastecimento - SDA-DDIV, 1998c. Cartão de identificação.

BRASIL. Ministério da Agricultura, do Abastecimento e da Reforma Agrária. Diário Oficial, n. 195, out. 1995. 112p. Suplemento.

CHANG, L.W.H.; MILLER, C.E. Pathway risk assessment: pink mealybug from the Caribbean. Washington: U.S. Department of Agriculture Animal and Plant Health Inspection Service, 1996. 61p.

KAIRO, M.T.K.; CROSS, A.E.; LOPEZ, V.F.; PETERKIN, D.D.; RAM, P. Rearing the hibiscus mealybug, Maconellicoccus hirsutus, and the parasitoid Anagyrus kamali Moursi. In: TECHNOLOGY TRANSFER WORKSHOP - BIOLOGICAL CONTROL OF PINK HIBISCUS MEALYBUG, 1998, St. Thomas. Handouts. St. Thomas: University of the Virgin Islands, 1998. $33 \mathrm{p}$.

KOGAN, M. Environmental impact of the introduction of quarantine pests. In: CONGRESSO BRASILEIRO DE ENTOMOLOGIA, 16., 1997, Salvador. Resumos. Salvador, BA, SEB/EMBARAPACNPMF, 1997. p.6.

MACONELLICOCCUS hirsutus (Green): simulation of potential geographic distribution using CLIMEX Simulation Model. 1998. In: TECHNOLOGY TRANSFER WORKSHOP - BIOLOGICAL CONTROL OF PINK HIBISCUS MEALYBUG, 1998, St. Thomas. Handouts. St Thomas: University of the Virgin Islands, 1998. 3p. 
MEYERDIRK, D. E.; WARKENLIN, R.; ATTAVIAN, B.; GERSABECK, E.; FRANCIS, A; ADAMS, M.; FRANCIS G. Biological control of pink hibiscus mealybyg: project manual. Riverdale: USDA-APHIS - PPQ, 1998. paginação irregular.

NARDO, E.A.B. De; TAMBASCO, F.J. O Brasil se prepara para combater a praga quarentenária Cochonilha-Rosada Maconellicoccus hirsutus (Green) (Hemiptera: Pseudococcidae). Informativo da Sociedade Entomológica do Brasil, v.23, n.2, p.5, ago. 1998.

NARDO, E.A.B. De; TAVARES, M.T.; SÁ, L.A.N. de; TAMBASCO. F.J. Perspectivas do controle biológico da praga quarentenária cochonilha-rosada no Brasil (Maconellicoccus hirsutus (GREEN) (Hemiptera: Pseudococcidae). Jaguariúna: Embrapa Meio Ambiente, 1999. 38p. (Embrapa Meio Ambiente. Documentos 2).

PERSAD, C. Public awareness and participation in biological control (Trinidad and Tobago): a case study. In: TECHNOLOGY TRANSFER WORKSHOP - BIOLOGICAL CONTROL OF PINK HIBISCUS MEALYBUG, 1998, St. Thomas. Handouts. St. Thomas: University of the Virgin Islands, 1998. 7p.

SILVA, J.R. da; SUMAN, R.; SILVA, O.L.R.; BORGATTO, D. de F. Cochonilha rosada, Maconellicocus hirsutus (Green), praga polífaga de hortaliças, fruteiras, ornamentais e essências florestais. Brasília: Ministério da Agricultura e do Abastecimento - Departamento de Defesa e Inspeção vegetal, 1997. 20p. (Série Alerta Quarentenário, 6).

STIBICK, J.N.L. New pest response guidelines: pink hibiscus mealybug, Maconellicoccus hirsutus. Washington, D.C.: U.S. Department of Agriculture - Animal and Plant Health Inspection Service, 1997. paginação irregular.

TAMBASCO, F.J. Cochonilha rosada está na fronteira com a Guiana. Fundecitrus, Fundo Paulista de Defesa da Citricultura, v. 13, n.89, p.15, out./nov. 1998. (Entrevista).

TAMBASCO, F.J.; NARDO, E.A.B. de. Atenção para a cochonilha rosada: colabore para evitar sua introdução no país ou denunciar focos de infestação. Jaguariúna: Embrapa Meio Ambiente/ Brasília: Ministério da 
Agricultura, 1998a. 11p.

TAMBASCO, F.J; NARDO, E.A.B. De. Cochonilha rosada pode invadir o Brasil. Informativo Embrapa Meio Ambiente, Jaguariúna, v.6, n.23, p.5, jul./set. 1998b. Entrevista.

THOMAS, A.; PETERS, T.; GRAHAM, P. Biological control of PMB in Grenada. In: TECHNOLOGY TRANSFER WORKSHOP BIOLOGICAL CONTROL OF PINK HIBISCUS MEALYBUG, 1998, St. Thomas. Handouts. St. Thomas: University of the Virgin Islands, 1998. 8p.

USDA. Animal and Plant Health Inspection Service. Pink hibiscus mealybug biological control program. Riverdale, 1997. 2p. (USDA-APHIS. Status Report, Aug. 6, 1997).

USDA. Animal and Plant Health Inspection Service. International Services \& Plant Protection and Quarantine. Pink hibiscus mealybyg (PHM) biological control program - United States Territories. In: TECHNOLOGY TRANSFER WORKSHOP - BIOLOGICAL CONTROL OF PINK HIBISCUS MEALYBUG, 1998, St. Thomas. Handouts. St. Thomas: University of the Virgin Islands. 1998. Não paginado. 